\title{
A Unidirectional Scheme for High-Fidelity Optical-Carrier Dissemination Using Phase-Modulation, Homodyne Coherent-Detection, and Frequency Entrainment
}

\author{
Ehsan Sooudi ${ }^{1,2}$, Fatima C. Garcia Gunning ${ }^{1,2}$, James O'Gorman ${ }^{3}$, Stylianos Sygletos ${ }^{4}$, Selwan K. Ibrahim ${ }^{5}$, \\ Andrew D. Ellis ${ }^{4}$, and Robert J. Manning ${ }^{1,2}$ \\ ${ }^{\text {I} P h o t o n i c ~ S y s t e m s ~ G r o u p, ~ T y n d a l l ~ N a t i o n a l ~ I n s t i t u t e, ~ L e e ~ M a l t i n g s, ~ C o r k, ~ I r e l a n d ~}$ \\ ${ }^{2}$ Department of Physics, University College Cork, Cork, Ireland \\ ${ }^{3}$ Xylophone Optics Ltd, Dublin, Ireland \\ ${ }^{4}$ Aston Institute of Photonic Technologies, Aston University, Birmingham, United Kingdom \\ ${ }^{5}$ FAZTech Research, Dublin, Ireland \\ ehsan.sooudi@tyndall.ie
}

\begin{abstract}
We report for the first time an ultra-stable optical-carrier dissemination technique for transmission over a $20 \mathrm{~km}$ unidirectional fibre link. The optical-linewidth of the recovered carrier matches closely that of the original carrier.

OCIS Codes: (120.4800) Optical standards and testing; (140.3520) Lasers, injection-locked; (120.5060) Phase modulation
\end{abstract}

\section{Introduction}

Accurate time and Optical Frequency Dissemination (OFD) of frequency standards based on optical transitions in laser cooled atoms and ions [1] have been widely demonstrated. Such dissemination allows wider usage of these standards for applications such as geodesy, fundamental physics tests, and comparison of frequency references. Demonstrations of direct and precise transmission of such optical references across installed optical fibre links with lengths of 10s [2] to 100s $\mathrm{km} \mathrm{[3,4]} \mathrm{have} \mathrm{been} \mathrm{reported.} \mathrm{Current} \mathrm{OFD} \mathrm{schemes} \mathrm{for} \mathrm{link} \mathrm{phase} \mathrm{noise} \mathrm{compensation}$ require both a dedicated (dark) bidirectional fibre link and amplifier(s) complicating deployment. Increasing demand for the practical availability of such references requires whatever dissemination scheme is deployed to be compatible with existing installed telecommunication fibre links. Such links are usually unidirectional and utilize optical/electrical (O/E) conversion of data channels, which means new approaches are needed if the present fibre network is to be used for precise and accurate frequency dissemination.

In this paper, we introduce and evaluate a novel scheme for dissemination of ultra-stable optical carriers which is deployable in a real telecommunications link. The scheme involves binary phase shift-keying (BPSK) modulation of the high-fidelity carrier and its subsequent recovery after transmission over a $20 \mathrm{~km}$ single-mode fibre (SMF) link, which includes dispersion compensating fibre (DCF). Carrier recovery from the carrier-less BPSK spectrum uses $\mathrm{O} / \mathrm{E}$ conversion and feed-forward based homodyne coherent detection [5]. The additional noise acquired by the signal due to transmission is cancelled by phase-locking of the recovered carrier to a stable CW laser through the injection-locking process. Initial experiments demonstrate excellent consistency of the original carrier linewidth with that of the injection-locked laser.

\section{Experiment}

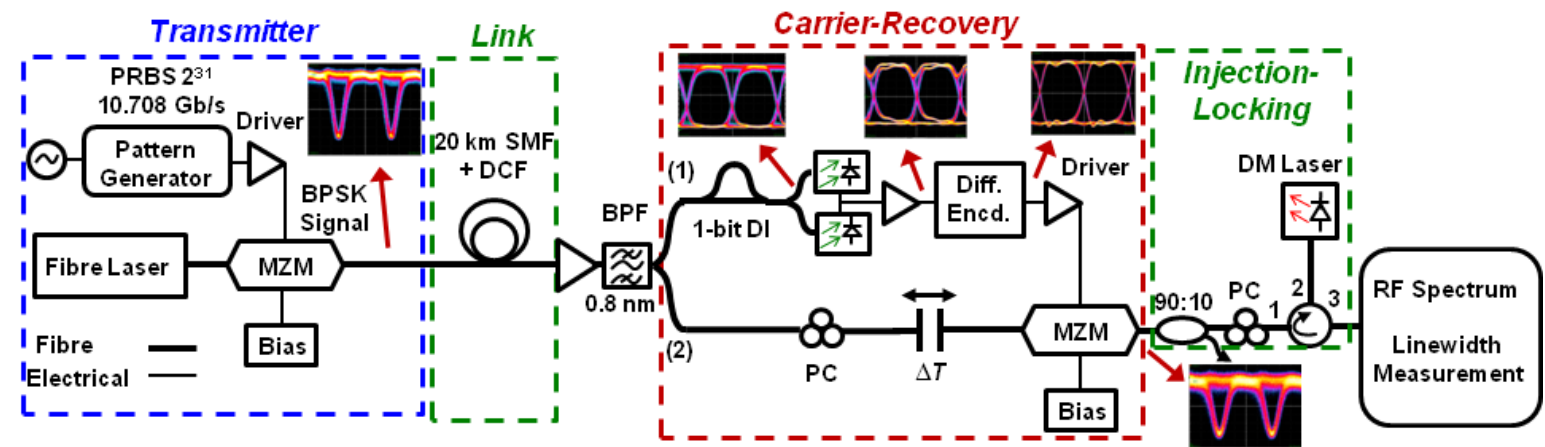

Fig. 1. Experimental arrangement for the dissemination scheme. List of acronyms: MZM: Mach-Zehnder Modulator, BPF: Band-pass filter, DI: Delayed interferometer, Diff. Encd. Differential Encoder, DM Laser: Discrete-Mode Laser, PC: Polarization controller, DCF: Dispersion compensating fibre, SMF: Single-mode fibre.

The diagram of our experimental arrangement is shown in Fig. 1. The set-up consists of three sections; a transmitter section followed by the fibre link, a carrier recovery section, and a laser injection locking section followed 
immediately by the linewidth measurement apparatus. The transmitter consists of an ultra-stable CW fibre laser (NP Photonics, Rock Source) which emits a high-fidelity low noise optical carrier, followed by a Mach-Zehnder modulator (MZM). The electrical signal (encoded data) originated from a pattern generator giving a Pseudo-Random Binary Sequence (PRBS, length: $2^{31}$ ), with bit-rate $10.709 \mathrm{~Gb} / \mathrm{s}$. A modulator driver generating a $V_{\pi}$ swing at the modulator, which was DC biased at null, created a binary phase-shift keyed (BPSK) modulation. This particular modulation format has $\pi$ phase shifts between 'zero' and 'one' bits. Its eye diagram is shown in Fig. 1. The signal is transmitted over a $20 \mathrm{~km}$ SMF link including DCF, followed by an optical amplifier and filter to compensate for the fibre loss. The carrier-recovery section generates the inverse of the original data pattern electronically and phase modulates the BPSK signal with this logically inverted pattern. This acts to cancel the deterministic phase modulation and so recover the original carrier. To achieve this, the incoming optical signal is split into two paths. In path (1) the BPSK optical signal is converted to an intensity modulated signal using the 1-bit delay interferometer (DI). The outputs from both ports of the DI are detected using balanced photo-diode detectors which drive a differential encoder via a limiting amplifier. The encoder generates the inverted data pattern electronically, which drives a MZM via a driver amplifier to remodulate the optical signal with this inverse pattern. A fine tuneable delay element in path (2) is used to match the timing of the optical signal arriving at the MZM to the generated data pattern in path (1). The now recovered carrier passes through a coupler and a polarization controller before being input to a CW discrete-mode (DM) [6] laser for injection-locking. The 10\% tap of the coupler is used to monitor the injection power. To minimize feedback, a circulator is placed before the laser, whose fibre pigtail is spliced to port 2 of the circulator. The laser's output is monitored through port 3 . The optical linewidth of the laser is measured using the self-heterodyne technique [7] and the RF spectrum is monitored to verify a stable-locking process.

The optical spectra of the BPSK modulated carrier (green), the recovered carrier (blue), and the injection-locked laser (red) are shown in Fig. 2(a). At optimum delay, a single-mode spectrum with $\sim 19$ dB side-mode suppression ratio (SMSR) was observed. The side modes in the spectrum of the recovered carrier originate from the null transitions of the second MZM used for the phase-modulation cancellation. Injection locking to the recovered carrier completely suppressed these side-bands. Stable-locking was confirmed by observing a clean RF spectrum with no frequency beating or amplitude fluctuations due to unstable locking. To prevent nonlinear instabilities, we kept the injection ratio low $(<-30 \mathrm{~dB})$ where a clear linewidth reduction from the $\mathrm{MHz}$ range to $\mathrm{kHz}$ range (shown in Fig. 2(b)) was achieved. The linewidth of injection-locked laser after $20 \mathrm{~km} \mathrm{SMF}$ was measured to be $5 \mathrm{kHz}$ (left inset Fig. 2(b)), close to that of the fibre laser (right inset), the original high-fidelity carrier.

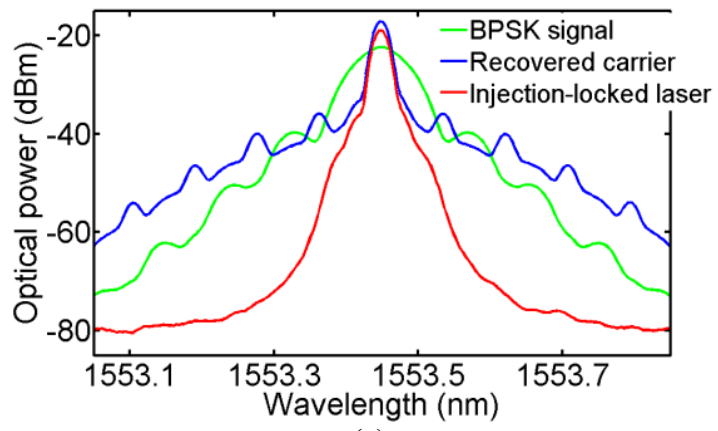

(a)

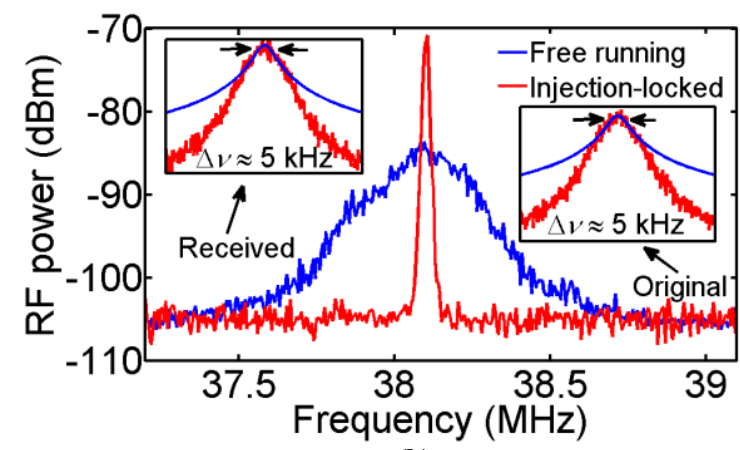

(b)

Fig. 2. (a). Optical spectra of the BPSK modulated signal (green), recovered carrier (blue), and injection-locked laser (red). (b). Self heterodyne optical beat-note of free-running DM laser (blue) and injection-locked to recovered carrier (red). The left inset shows received carrier's lineshape (after $20 \mathrm{~km} \mathrm{SMF)} \mathrm{fitted} \mathrm{with} \mathrm{Lorentzian} \mathrm{distribution} \mathrm{(optical} \mathrm{linewidth} \approx 5 \mathrm{kHz}$ ). Right inset shows that of the original carrier.

\section{Conclusion}

We have investigated in detail a novel dissemination technique for ultra-stable optical-carriers which is compatible with existing (non-bidirectional) telecommunication fibre links. With transmission over a $20 \mathrm{~km}$ fibre link, we see excellent agreement between the optical-linewidths of original and received carriers. On-going systematic measurements of phase-noise and Allan deviation will determine the ultimate limitations of this scheme.

This work was supported by European Space Agency (ESA) under Contract no. 4000107786/13/D/MRP.

\section{References}

[1] T. Rosenband, et al., Science 319, 1808 (2008).

[2] S. M. Foreman, et al., Phys. Rev. Lett., vol. 99, 2007.

[3] N. Newbury, P. Williams, and W. Swann, Opt. Lett. 32, 2007.

[4] K. Predehl, et al., Science, vol. 336, 2012, 2012.

[5] S. K. Ibrahim, et al. Opt. Express, vol. 19, no. 9, 2011.
[6] S. O'Brien, et al., IEEE JSTQE, vol.17, no.6, 2011.

[7] D. Derickson, Ed., Fiber Optic Test and Measurement. Upper Saddle River: NJ: Prentice-Hall, 1998. 